\title{
Logarithmic Normal Population Distribution of Parameter of Joint Fiducial Region Estimation \\ Xiuzhen $\mathrm{Li}^{1,{ }^{1},{ }^{*} \text {, Yanying } \mathrm{Ma}^{1} \text { and Chunguang Huang }}{ }^{2}$ \\ ${ }^{1}$ Media and Mathematical institute Jiliin Engineering Normal University JiLin China \\ ${ }^{2} \mathrm{NO} .11$ High school of Changchun JiLin China \\ a1060138576@qq.com
}

Keywords: Lognormal population; Fiducial distribution; Combination fiducial region

\begin{abstract}
According to the lognormal distribution and normal distribution relationship of logarithmic normal distribution is given two parameters interval estimation of two lognormal distribution parameters and Combination fiducial region estimation.
\end{abstract}

\section{The Definition of Lognormal Distribution}

Lognormal distribution is named because of the logarithmic obey normal distribution, the so called lognormal distribution. Definition: a population X obey the lognormal distribution, the distribution density function is

$$
f\left(x ; \mu, \sigma^{2}\right)= \begin{cases}\frac{1}{\sqrt{2 \pi} \sigma x} e^{-\frac{(\ln x-\mu)^{2}}{2 \sigma^{2}}} & x>0 \\ 0 & x \leq 0\end{cases}
$$

Among them $-\infty<\mu<\infty, \sigma^{2}>0$

\section{Establish Function Model, the Induction of Faith Distribution}

Definition: set to simple random sample $X_{1}, X_{2}, \ldots X_{n}$ from lognormal population $\mathrm{X}$, to remember

$$
\begin{aligned}
& \overline{\ln X}=\frac{1}{n} \sum_{i=1}^{n} \ln X_{i} \\
& Q^{2}=\sum_{i=1}^{n}\left(\ln X_{i}-\overline{\ln X}\right)^{2}
\end{aligned}
$$

According to the lognormal distribution and normal distribution and the relationship between basic theorem, the sampling distribution has the following lemma:

Lemma 1.

(1) $\overline{\ln X}$ 与 $Q^{2}$ independent of each other

(2) $\sqrt{n(n-1)} \frac{\overline{\ln X}-\mu}{Q} \sim \mathrm{t}(\mathrm{n}-1)$

(3) $\frac{Q^{2}}{\sigma^{2}} \sim \chi^{2}(\mathrm{n}-1)$

(4) $\left(\overline{\ln X}, Q^{2}\right)$ is a sufficient $\operatorname{statistic}\left(\mu, \sigma^{2}\right)$

Due to the sufficient statistic of $\left(\overline{\ln X}, Q^{2}\right)$ is $\left(\overline{\ln X}, Q^{2}\right)$, So we based on the principle of sufficiency, consider the combination of $\left(\mu, \sigma^{2}\right)$ by $\left(\overline{\ln X}, Q^{2}\right)$ combination fiducial region 
estimation .Because $\overline{\ln X} \sim \mathrm{N}\left(\mu, \frac{\sigma^{2}}{n}\right)$, By the lemma (1.1) and (3) to know $\frac{Q^{2}}{\sigma^{2}} \sim \chi^{2}$ (n-1), Again by lemma (1.1) and (1) $\overline{\ln X}$ 与 $Q^{2}$ independent of each other. If remember the error variables $e_{1} \sim$ $\mathrm{N}(0,1), e_{2} \sim \chi^{2}(\mathrm{n}-1)$, and $e_{1}, e_{2}$ are independent of each other. The function model is set up

$$
\left\{\begin{array}{c}
\overline{\ln X}=\mu+\frac{\sigma}{\sqrt{n}} \cdot e_{1} \\
Q^{2}=\sigma^{2} \cdot e_{2}
\end{array}\right.
$$

One $\left(\overline{\ln X}, Q^{2}\right)$ as the sample observations; $\left(\mu, \sigma^{2}\right)$ for the unknown parameters; $\left(e_{1}, e_{2}\right)$ as the error variable. Transposition function model by on (3)

This can be the joint distribution of faith of $\left(\mu, \sigma^{2}\right)$. In a sample observation value $X$, thus has the $\overline{\ln X}$ and $Q^{2}$, induced by function model the combination fiducial distribution of $\left(\mu, \sigma^{2}\right)$. Due to the $e_{1} \sim \mathrm{N}(0,1), e_{2} \sim \chi^{2}(\mathrm{n}-1)$, and $e_{1}$ and 与 $e_{2}$ are independent of each other. Therefore the joint distribution density function of $\left(e_{1}, e_{2}\right)$ is

$$
\mathrm{h}\left(e_{1}, e_{2}\right)=\frac{1}{\sqrt{2 \pi}} e^{-\frac{e_{1}^{2}}{2}} \cdot \frac{1}{2^{\frac{n-1}{2}} \Gamma\left(\frac{n-1}{2}\right)} e^{\frac{n-1}{2}-1} \cdot e^{-\frac{e_{2}}{2}} \text { among them }-\infty<e_{1}<\infty, e_{2}>0
$$

Due to $e_{1}=\frac{\sqrt{n(\overline{\ln x}-\mu)}}{\sigma}, e_{2}=\frac{Q^{2}}{\sigma^{2}}$ according to random vector transform formula: from

$\left(e_{1}, e_{2}\right)$ to $\left(\mu, \sigma^{2}\right)$ transformation, the transformation of the absolute value of the jacobian for

$$
|J|=\left\|\begin{array}{ll}
\frac{\partial e_{1}}{\partial \mu} & \frac{\partial e_{1}}{\partial \sigma^{2}} \\
\frac{\partial e_{2}}{\partial \mu} & \frac{\partial e_{2}}{\partial \sigma^{2}}
\end{array}\right\|=\left\|\begin{array}{cc}
\frac{-\sqrt{n}}{\sigma} & \frac{-1}{2} \sqrt{n}(\overline{\ln x}-\mu)\left(\sigma^{2}\right)^{-\frac{3}{2}} \\
0 & -Q^{2} \frac{1}{\sigma^{4}}
\end{array}\right\|=Q^{2} \frac{\sqrt{n}}{\sigma^{5}}
$$

In $e_{1}, e_{2}$ available $\left(\mu, \sigma^{2}\right)$ combination fiducial distribution density function is

$$
\begin{aligned}
& \mathrm{g}\left(\mu, \sigma^{2}\right)=\frac{1}{\sqrt{2 \pi}} e^{-\frac{\mathrm{n}(\overline{\ln x}-\mu)^{2}}{2 \sigma^{2}}} \cdot \frac{1}{2^{\frac{n-1}{2}} \Gamma\left(\frac{n-1}{2}\right)}\left(\frac{Q^{2}}{\sigma^{2}}\right)^{\frac{n-3}{2}} \cdot e^{-\frac{Q^{2}}{2 \sigma^{2}}} Q^{2} \frac{\sqrt{n}}{\sigma^{5}} \\
& =\frac{\sqrt{n}}{\sqrt{2 \pi} \sigma} \exp \left[-\frac{n(\overline{\ln x}-\mu)^{2}}{2 \sigma^{2}}\right] \frac{\left(Q_{2}\right)^{\frac{n-1}{2}}}{\left(2 \sigma^{2}\right)^{\frac{n-1}{2}} \Gamma\left(\frac{n-1}{2}\right)} \cdot e^{-\frac{Q^{2}}{2 \sigma^{2}}}
\end{aligned}
$$

(5) compared with (7), it is very similar. (3.5) from 0 to $\infty$ to $\sigma^{2}$ points, can be marginal beliefs about $\mu$ distribution; (3.5) from $-\infty$ to $\infty$ to $\mu$ points, can be on $\sigma^{2}$ the marginal distribution of religion, which is available in principle.

We also can use another way: we also can be directly by (1.3), respectively, the marginal fiducial of $\mu$ and $\sigma^{2}$ distribution is deduced. $\mu$ is derived under the marginal fiducial distribution:

By (3) and the definition of the $\mathrm{t}$ distribution we can derive

$$
\sqrt{(n-1)} \frac{\sqrt{n}(\mu-\overline{\ln X})}{Q}=-\sqrt{n-1} \cdot \frac{e_{1}}{\sqrt{e_{2}}} \sim \mathrm{t}(\mathrm{n}-1)
$$


Therefore the marginal fiducial distribution of $\mu$ is $\mathrm{t}$ distribution.

Under the marginal fiducia distribution of $\sigma^{2}$ is derived:

By (3) can also $\frac{\sigma^{2}}{Q^{2}}=\frac{1}{e_{2}}, e_{2} \sim \chi^{2}(\mathrm{n}-1)$

So the marginal fiducial distribution of $\sigma^{2}$ is reversal $\chi^{2}$ distribution, the distribution density function is

$$
\propto \exp \left\{-\frac{Q^{2}}{2 \sigma^{2}}\right\}\left(\sigma^{2}\right)^{-\frac{n+1}{2}} \quad \sigma^{2}>0
$$

\section{Fiducial Region (interval) Estimates Is Given}

To solve the fiducial interval of $\mu$ :

Fiducial distribution of $\mu$ Given by (6) for $\mu$ interval estimation for fiducial level $1-\alpha$ is

$\left[\overline{\ln X}-\frac{s}{\sqrt{n}} t_{1-\frac{\alpha}{2}}(n-1), \overline{\ln X}+\frac{s}{\sqrt{n}} t_{1-\frac{\alpha}{2}}(n-1)\right]$

Because the $t$ distribution is unimodal symmetric distribution, so the fiducial interval length is the shortest, is an optimal fiducial interval.

To solve the fiducial interval of $\sigma^{2}$ :

$\chi_{\frac{\alpha}{2}}^{2}(n-1) \leq e_{2} \leq \chi_{1-\frac{\alpha}{2}}^{2}(n-1)$ Inequality, available $\sigma^{2}$ for the $1-\alpha$ fiducial level fiducial interval $\left[\frac{(n-1) S^{2}}{\chi_{1-\frac{\alpha}{2}}^{2}(n-1)}, \frac{(n-1) S^{2}}{\chi_{\frac{\alpha}{2}}^{2}(n-1)}\right]$ (Note: this is not the optimum interval estimation.)

All available the following theorem

Theorem 1.1: set $\mathrm{X}$ to lognormal population distribution $\mathrm{N}\left(\mu, \sigma^{2}\right)$, the distribution density function is

$$
f\left(x ; \mu, \sigma^{2}\right)= \begin{cases}\frac{1}{\sqrt{2 \pi} \sigma x} e^{-\frac{(\ln x-\mu)^{2}}{2 \sigma^{2}}} & x>0 \\ 0 & x \leq 0\end{cases}
$$

among them $-\infty<\mu<\infty, \sigma^{2}>0$ is parameter, then the

combination fiducial region of fiducial level $1-\alpha$ for $\left(\mu, \sigma^{2}\right)$ is

$$
\mathrm{G}=\left\{\left(\mu, \sigma^{2}\right) \mid(\overline{\ln X}-\mu)^{2} \leq \frac{\sigma^{2}}{n} a_{2}{ }^{2}, \quad \frac{Q^{2}}{b_{2}}<\sigma^{2}<\frac{Q^{2}}{b_{1}}\right\} \text { The calculation formula for the area of the }
$$
region $\mathrm{G}$ is

$$
S_{G}=2 \int_{\frac{(n-1) s^{2}}{b_{2}}}^{\frac{(n-1) s^{2}}{b_{1}}} \int \frac{\sigma}{\ln x} a_{2} d \mu d \sigma^{2}=2 \int_{\frac{(n-1) s^{2}}{b_{2}}}^{\frac{(n-1) s^{2}}{b_{1}}} \frac{\sigma}{\sqrt{n}} a_{2} d \sigma^{2}=\frac{4 a_{2}}{3 \sqrt{n}}(n-1)^{\frac{3}{2}} s^{3}\left(b_{1}^{-\frac{3}{2}}-b_{2}^{-\frac{3}{2}}\right)
$$

(For a given level of faith $1-\alpha$, to select suitable $a_{1}, a_{2}, b_{1}, b_{2}$ ) 


\section{Acknowledgements}

Jilin provincial department of education project (2016): research and applications of mineral prediction methods based on grey theory.

\section{References}

[1] Mao Shi-song, Wang Jing-long, Pu Xiao-long. Advanced Mathematical Statistics [M]. higher education publishing house, 1998

[2] Chen Xi-ru., Mathematical Statistics Introduction [M]. science press, 1981.

[3] Chen Guang-shu. Combination confidence region of lognormal population distribution [ J]

[4] Dawid A P and Stone M. The functional-model basis of fiducial inference(with discussion).Ann Statist, 1982,10:01054-1074

[5]Gunnar B. Probability and statistics Theory and applicationas. Newyork springer, 1989. 